\title{
ON SUBCLASSES OF UNIFORMLY CONVEX FUNCTIONS AND CORRESPONDING CLASS OF STARLIKE FUNCTIONS
}

\author{
R. BHARATI, R. PARVATHAM AND A. SWAMINATHAN
}

\begin{abstract}
We determine a sufficient condition for a function $f(z)$ to be uniformly convex of order $\alpha$ that is also necessary when $f(z)$ has negative co-efficients. This enables us to express these classes of functions in terms of convex functions of particular order. Similar results for corresponding classes of starlike functions are also obtained. The convolution condition for the above two classes are discussed.
\end{abstract}

\section{Introduction}

Let $S$ be the class of functions

$$
f(z)=z+\sum_{n=2}^{\infty} a_{n} z^{n}
$$

that are analytic and univalent in the unit disk $E=\{z:|z|<1\}$. Let $S^{*}(\alpha)$ and $K(\alpha)$ denote the subclasses of functions $f(z)$ in $S$ such that $\operatorname{Re}\left\{\frac{z f^{\prime}(z)}{f(z)}\right\}>\alpha$ and $\operatorname{Re}\{1+$ $\left.\frac{z f^{\prime \prime}(z)}{f^{\prime}(z)}\right\}>\alpha, 0 \leq \alpha<1$, respectively. $S^{*}(0)=S^{*}$ and $K(0)=K$ denote the ordinary class of starlike functions and convex functions respectively. Goodman [1] defined the following subclass of $K$.

Definition 1.1.([1]) A function $f(z)$ is uniformly convex in $E$ if $f(z)$ is in $K$ and has the property that for every circular arc $\gamma$ contained in $E$, with centre $\varepsilon$ also in $E$, the arc $f(\gamma)$ is convex.

Ronning [2] found a more applicable one variable analytic characterization for the above class denoted by UCV.

Definition 1.2.([2]) A function $f(z)=z+\sum_{n=2}^{\infty} a_{n} z^{n}$ is in UCV if and only if

$$
\operatorname{Re} 1+\frac{z f^{\prime \prime}(z)}{f^{\prime}(z)} \geq\left|\frac{z f^{\prime \prime}(z)}{f^{\prime}(z)}\right|, z \in E
$$

Received Jun. 8, 1996; revise April 19, 1996.

1991 Mathematics Subject Classification. 30C45.

Key words and phrases. Univalent, starlike, uniformly convex. 
Definition 1.3.([5]) Let $T$ be the subfamily of $S$ consisting of functions of the form

$$
f(z)=z-\sum_{n=2}^{\infty} a_{n} z^{n}, \quad a_{n} \geq 0, z \in E .
$$

Let $T^{*}(\alpha)$ and $C^{*}(\alpha)$ be the subfamily of functions in $T$ that are starlike of order $\alpha$ and convex of order $\alpha$ respectively for $0 \leq \alpha<1$. Silverman [5] gave a co-efficient characterization for these classes.

Theorem A.([5]) A function $f(z)=z-\sum_{n=2}^{\infty} a_{n} z^{n}, a_{n} \geq 0$ is in $T^{*}(\alpha)$ if and only if $\sum_{n=2}^{\infty}\left(\frac{n-\alpha}{1-\alpha}\right) a_{n} \leq 1$ and is in $C^{*}(\alpha)$ if and only if $\sum_{n=2}^{\infty} \frac{n(n-\alpha)}{1-\alpha} a_{n} \leq 1$.

This led to the following sharp distortion bounds and extreme points.

Theorem B.([5]) (i) If $f(z) \in T^{*}(\alpha)$, then

$$
\begin{gathered}
r-\frac{1-\alpha}{2-\alpha} r^{2} \leq|f(z)| \leq r+\frac{1-\alpha}{2-\alpha} r^{2} \\
1-\frac{2(1-\alpha)}{2-\alpha} r \leq\left|f^{\prime}(z)\right| \leq 1+\frac{2(1-\alpha)}{2-\alpha} r
\end{gathered}
$$

and the extreme points are given by

$$
f_{1}(z)=z, f_{n}(z)=z-\frac{1-\alpha}{n-\alpha} z^{n} \quad(n=2,3, \cdots) .
$$

(ii) If $f(z) \in C^{*}(\alpha)$, then

$$
\begin{gathered}
r-\frac{1-\alpha}{2(2-\alpha)} r^{2} \leq|f(z)| \leq r+\frac{1-\alpha}{2(2-\alpha)} r^{2} \\
1-\frac{1-\alpha}{2-\alpha} r \leq\left|f^{\prime}(z)\right| \leq 1+\frac{1-\alpha}{2-\alpha} r,
\end{gathered}
$$

and the extreme points are

$$
f_{1}(z)=z, f_{n}(z)=z-\frac{1-\alpha}{n(n-\alpha)} z^{n} \quad(n=2,3, \cdots) .
$$

Ronning [3] introduced the class of functions $S_{p}$ such that $f \in \mathrm{UCV} \Leftrightarrow z f^{\prime} \in S_{p}$.

Ronning generalized the class UCV and $S_{p}$ by introducing a parameter $\alpha$ in the following way.

Definition 1.4. ([4]) A function $f(z)$ is in $S_{p}(\alpha)$ if $f(z)$ satisfies the analytic characterization

$$
\left|\frac{z f^{\prime}(z)}{f(z)}-1\right| \leq \operatorname{Re} \frac{z f^{\prime}(z)}{f(z)}-\alpha
$$

and $f(z)$ is in $\operatorname{UCV}(\alpha)$ if and only if $z f^{\prime}(z)$ is in $S_{p}(\alpha)$. 
Therefore for the class $S_{p}(\alpha)$ [2] we get a domain whose boundary is a parabola with vertex $w=\frac{1+\alpha}{2}$. We see that for all $\alpha \in[-1,1)$ we have $S_{p}(\alpha) \subset S^{*}$ and $S_{p}(\alpha) \not \subset S$ for $\alpha<-1$. Hence we observe that $\operatorname{UCV}(\alpha) \subset K$ for $\alpha \geq-1$.

Ronning [4] generalized the class $S_{p}$ in a different manner than (1.7).

Definition 1.5. ([4]) A function $f(z)=z+\sum_{n=2}^{\infty} a_{n} z^{n}$ is in the class $P(\alpha, \beta)$ if $f(z)$ satisfies the analytic characterization

$$
\left|\frac{z f^{\prime}(z)}{f(z)}-(\alpha+\beta)\right| \leq \operatorname{Re} \frac{z f^{\prime}(z)}{f(z)}+\alpha-\beta \quad 0<\alpha<\infty, 0 \leq \beta<1, \quad z \in E .
$$

This means that $\frac{z f^{\prime}(z)}{f(z)}$ for $f(z) \in p(\alpha, \beta)$ and $z \in E$ lies in that portion of the plane which contains $w=1$ and is bounded by the parabola $y^{2}=4 \alpha(x-\beta)$. We observe that $S_{p}=P\left(\frac{1}{2}, \frac{1}{2}\right)$. Since $P(\alpha, \beta) \subset P(\alpha, 0)$ it seems to be most interesting in this context to study the classes where $\beta=0$. For simplicity of notation, we define $P(\alpha)=P(\alpha, 0)$ and hence we have

$$
p(\alpha)=\left\{f(z) \in S ; \quad\left|\frac{z f^{\prime}(z)}{f(z)}-\alpha\right| \leq \operatorname{Re} \frac{z f^{\prime}(z)}{f(z)}+\alpha \quad z \in E, 0<\alpha<\infty\right\} .
$$

In this note we obtain co-efficient characterization for some subclasses of UCV $(\alpha)$ and $S_{p}(\alpha)$. In section 2 , we define some subclasses of $\operatorname{UCV}(\alpha), S_{p}(\alpha)$ and $P(\alpha)$ with negative co-efficients for $f(z)$. In section 3 , we obtain some convolution results for these classes of functions.

\section{Co-efficient Characterization}

Definition 2.1. Let $\operatorname{UCT}(\alpha)$ be the class of functions $f(z)=z-\sum_{n=2}^{\infty} a_{n} z^{n}, a_{n} \geq 0$ satisfy the condition

$$
\left|\frac{z f^{\prime \prime}(z)}{f^{\prime}(z)}\right| \leq \operatorname{Re}\left\{1+\frac{z f^{\prime \prime}(z)}{f^{\prime}(z)}-\alpha\right\}, \quad z \in E .
$$

Definition 2.2. Let $S_{p} T(\alpha)$ be the class of functions $f(z)=z-\sum_{n=2}^{\infty} a_{n} z^{n}, a_{n} \geq 0$ satisfy the condition

$$
\left|\frac{z f^{\prime}(z)}{f^{\prime}(z)}-1\right| \leq \operatorname{Re}\left\{\frac{z f^{\prime}(z)}{f(z)}\right\}-\alpha, \quad z \in E .
$$

Now we obtain a sufficient condition for $f(z)$ in $\operatorname{UCV}(\alpha)$.

Theorem 2.1. If

$$
\sum_{n=2}^{\infty}(2 n-1-\alpha) n\left|a_{n}\right| \leq 1-\alpha
$$


then $f(z)=z+\sum_{n=2}^{\infty} a_{n} z^{n}, z \in E$ is in $U C V(\alpha)$.

Proof. In view of the definition of $\operatorname{UCV}(\alpha)$ it is sufficient if we verify the condition

$$
\left|\frac{z f^{\prime \prime}(z)}{f^{\prime}(z)}\right| \leq \operatorname{Re} \frac{z f^{\prime \prime}(z)}{f^{\prime}(z)}+1-\alpha .
$$

We have

$$
\begin{aligned}
\left|\frac{z f^{\prime \prime}(z)}{f^{\prime}(z)}\right|-R e \frac{z f^{\prime \prime}(z)}{f^{\prime}(z)} & \leq 2\left|\frac{z f^{\prime \prime}(z)}{f^{\prime}(z)}\right| \\
& \leq \frac{\sum_{n=2}^{\infty} 2 n(n-1)\left|a_{n}\right||z|^{n-1}}{1-\sum_{n=2}^{\infty} n\left|a_{n}\right||z|^{n-1}} \\
& \leq \frac{\sum_{n=2}^{\infty} 2 n(n-1)\left|a_{n}\right|}{1-\sum_{n=2}^{\infty} n\left|a_{n}\right|}
\end{aligned}
$$

The above expression is bounded by $1-\alpha$ if and only if (2.3) is satisfied and the proof is complete.

Now we show that the sufficient condition of Theorem 2.1 for $\operatorname{UCV}(\alpha)$ is also a necessary condition for $\mathrm{UCT}(\alpha)$.

Theorem 2.2. Let $f(z)=z-\sum_{n=2}^{\infty} a_{n} z^{n}, a_{n} \geq 0$ then $\sum_{n=2}^{\infty} n(2 n-1-\alpha) a_{n} \leq 1-\alpha$ if and only if $f(z)$ is in $U C T(\alpha)$.

Proof. In view of Theorem 2.1 we need only show that $f(z)$ in $\operatorname{UCT}(\alpha)$ satisfies the co-efficient inequality.

If $f(z) \in \operatorname{UCT}(\alpha)$ and $z$ is real, then the definition of $\operatorname{UCT}(\alpha)$ yields

$$
1-\frac{\sum_{n=2}^{\infty} n(n-1) a_{n} z^{n-1}}{1-\sum_{n=2}^{\infty} n a_{n} z^{n-1}}-\alpha \geq \frac{\sum_{n=2}^{\infty} n(n-1) a_{n} z^{n-1}}{1-\sum_{n=2}^{\infty} n a_{n} z^{n-1}}
$$

. Let $z \rightarrow 1$ along the real axis, then we get

$$
(1-\alpha) \geq \frac{2 \sum_{n=2}^{\infty} n(n-1) a_{n}}{1-\sum_{n=2}^{\infty} n a_{n}}
$$

or

$$
(1-\alpha) 1-\sum_{n=2}^{\infty} n a_{n} \geq 2 \sum_{n=2}^{\infty} n(n-1) a_{n}
$$

which gives the required result.

Corollary 2.1. $U C T(\alpha)=C^{*}\left(\frac{1+\alpha}{2}\right)$.

Proof. From Theorem 1.2 we get the functions in $\operatorname{UCT}(\alpha)$ satisfy the condition

$$
\sum_{n=2}^{\infty} \frac{n(2 n-1-\alpha)}{1-\alpha} a_{n} \leq 1
$$


which is equivalent to saying that

$$
\sum_{n=2}^{\infty} \frac{n\left[n-\left(\frac{1+\alpha}{2}\right)\right]}{1-\left(\frac{1+\alpha}{2}\right)} \leq 1
$$

The above expression in comparison with Theorem $A$ gives the result.

Corollary 2.2. If $f(z) \in U C T(\alpha)$, then

$$
\begin{gathered}
r-\frac{1-\alpha}{2(3-\alpha)} r^{2} \leq|f(z)| \leq r+\frac{1-\alpha}{2(3-\alpha)} r^{2} \\
1-\frac{1-\alpha}{3-\alpha} r \leq\left|f^{\prime}(z)\right| \leq 1+\frac{1-\alpha}{3-\alpha} r
\end{gathered}
$$

and the extreme points are

$$
f_{1}(z)=z, f_{n}(z)=z-\frac{1-\alpha}{n(2 n-1-\alpha)} z^{n}, \quad n=2,3, \cdots .
$$

Proof. Since the distortion theorems are extreme points are available for $C^{*}(\alpha)$ in Theorem B, the distortion theorems and extreme points are immediately available by applying Corollary 2.1 .

Definition 2.3. Let $U C T(\alpha, \beta)$ be the class of functions $f(z)=z-\sum_{n=2}^{\infty} a_{n} z^{n}$, $a_{n}>0$ that satisfy the condition

$$
\operatorname{Re}\left\{1+\frac{z f^{\prime \prime}(z)}{f^{\prime}(z)}\right\} \geq \alpha\left|\frac{z f^{\prime \prime}(z)}{f^{\prime}(z)}\right|+\beta, \quad \alpha \geq 0, \beta \geq 0 .
$$

We write $U C T(1, \beta)=U C T(\beta)$ and observe that $U C T(0, \beta)=C^{*}(\beta)$.

Theorem 2.3. A function $f(z)=z-\sum_{n=2}^{\infty} a_{n} z^{n}$ is in $U C T(\alpha, \beta)$ if and only if

$$
\sum_{n=2}^{\infty}[n(1+\alpha)-(\alpha+\beta)] n a_{n} \leq 1-\beta \text {. }
$$

Proof. Proceeding as in Theorem 2.2, we get that, for $f(z) \in \operatorname{UCT}(\alpha, \beta)$ it is sufficient to verify the co-efficient inequality.

Therefore for $z$ real, we get

$$
1-\frac{\sum_{n=2}^{\infty} n(n-1) a_{n} z^{n-1}}{1-\sum_{n=2}^{\infty} n a_{n} z^{n-1}} \geq \alpha \frac{\sum_{n=2}^{\infty} n(n-1) a_{n} z^{n-1}}{1-\sum_{n=2}^{\infty} n a_{n} z^{n-1}}+\beta .
$$

Let $z \rightarrow 1$ along the real axis, which gives

$$
(1-\beta) \geq(1+\alpha) \frac{\sum_{n=2}^{\infty} n(n-1) a_{n}}{1-\sum_{n=2}^{\infty} n a_{n}}
$$


which is equivalent to

$$
(1-\beta)\left\{1-\sum_{n=2}^{\infty} n a_{n}\right\} \geq(1+\alpha) \sum_{n=2}^{\infty} n(n-1) a_{n}
$$

which gives the required result and the proof is complete.

Corollary 2.3. $U C T(\alpha, \beta)=C^{*}\left(\frac{\alpha+\beta}{1+\alpha}\right)$.

Proof. From Theorem 2.3 for $f(z)$ in $\operatorname{UCT}(\alpha, \beta)(2.5)$ can be written in an equivalent form

$$
\sum_{n=2}^{\infty}\left[\frac{n-\left(\frac{\alpha+\beta}{1+\alpha}\right)}{1-\left(\frac{\alpha+\beta}{1+\alpha}\right)}\right] n a_{n} \leq 1
$$

which together with Theorem A gives the result.

Hence the distortion theorems and extreme points are immediately available for $f(z)$ in $\operatorname{UCT}(\alpha, \beta)$.

Corollary 2.4. If $f(z) \in U C T(\alpha, \beta)$, then

$$
\begin{gathered}
r-\frac{1-\beta}{2(2+\alpha-\beta)} r^{2} \leq|f(z)| \leq r+\frac{1-\beta}{2(2+\alpha-\beta)} r^{2} \\
1-\frac{1-\beta}{2+\alpha-\beta} r \leq\left|f^{\prime}(z)\right| \leq 1+\frac{1-\beta}{2+\alpha-\beta} r
\end{gathered}
$$

and the extreme points are

$$
f_{1}(z)=z, f_{n}(z)=z-\frac{1-\beta}{n[n(1+\alpha)-(\alpha+\beta)]} z^{n}, \quad n=2,3, \cdots .
$$

Remark. In [5] it is given that

$$
C^{*}(\alpha) \subset T^{*}\left(\frac{2}{3-\alpha}\right)
$$

Therefore we get the

implies that

$$
\mathrm{UCT}(\alpha, \beta)=C^{*}\left(\frac{\alpha+\beta}{1+\alpha}\right)
$$

$$
\mathrm{UCT}(\alpha, \beta) \subset T^{*}\left(\frac{2 \alpha+2}{2 \alpha+3-\beta}\right) .
$$

In particular $\operatorname{UCT}(1,0)=\mathrm{UCT}$ with $f(z)=z-\frac{z^{2}}{6}$ being sharp.

Now let us draw our attention to the class $S_{p}(\alpha)$ and $S_{p} T(\alpha)$ and find their co-efficient criterion. 
Theorem 2.4. If $\sum_{n=2}^{\infty}(2 n-1-\alpha)\left|a_{n}\right| \leq 1-\alpha$ then $f(z)=z+\sum_{n=2}^{\infty} a_{n} z^{n}$ is in $S_{p}(\alpha)$.

Proof. By Alexander type theorem, we get that $f(z) \in \operatorname{UCV}(\alpha) \Longleftrightarrow z f^{\prime}(z) \in S_{p}(\alpha)$. Therefore by replacing the co-efficient $\left|a_{n}\right|$ in Theorem 2.1 by $\frac{\left|a_{n}\right|}{n}$, the required result is obtained.

Remark. For $\alpha=0$, the above result gives the co-efficient characterization which is sufficient condition for a function to be in the class $S_{p}$.

Since $f(z) \in \operatorname{UCT}(\alpha) \Longleftrightarrow z f^{\prime}(z) \in S_{p} T(\alpha)$ and $f(z) \in \operatorname{UCT}(\alpha, \beta) \Longleftrightarrow z f^{\prime}(z) \epsilon$ $S_{p} T(\alpha, \beta)$, the co-efficient $a_{n}$ in Theorem 2.2 and Theorem 2.3 can be replaced by $\frac{a_{n}}{n}$ to get the required result for $S_{p} T(\alpha)$ and $S_{p} T(\alpha, \beta)$ respectively. Hence we state the results directly.

Theorem 2.5. $f(z)=z-\sum_{n=2}^{\infty} a_{n} z^{n}, a_{n} \geq 0$ is in $S_{p} T(\alpha)$ if and only if $\sum_{n=2}^{\infty}(2 n-$ $1-\alpha) a_{n} \leq 1-\alpha$.

Theorem 2.6. $f(z)=z-\sum_{n=2}^{\infty} a_{n} z^{n}, a_{n} \geq 0$ is in $S_{p} T(\alpha, \beta)$ if and only if $\sum_{n=2}^{\infty}[n(1+\alpha)-(\alpha+\beta)] a_{n} \leq 1-\beta$.

Remark. Comparing Theorem A with Theorem 2.5 and Theorem 2.6 we get that

(i) $S_{p} T(\alpha)=T^{*}\left(\frac{1+\alpha}{2}\right)$

(ii) $S_{p} T(\alpha, \beta)=T^{*}\left(\frac{\alpha+\beta}{1+\alpha}\right)$.

Thus distortion bounds and extreme points of $S_{p} T(\alpha)$ and $S_{p} T(\alpha, \beta)$ are easily available and we state them using Theorem B.

Corollary 2.5. If $f(z) \in S_{p} T(\alpha)$, then

$$
\begin{gathered}
r-\frac{1-\alpha}{3-\alpha} r^{2} \leq|f(z)| \leq r+\frac{1-\alpha}{3-\alpha} r^{2} \\
1-\frac{2(1-\alpha)}{3-\alpha} r \leq\left|f^{\prime}(z)\right| \leq 1+\frac{2(1-\alpha)}{3-\alpha} r
\end{gathered}
$$

and the extreme points are

$$
f_{1}(z)=z \text { and } f_{n}(z)=z-\frac{1-\alpha}{2 n-1-\alpha} z^{n}, \quad n=2,3, \cdots .
$$

Corollary 2.6. If $f(z) \in S_{p} T(\alpha, \beta)$ then

$$
\begin{aligned}
& r-\frac{1-\beta}{2+\alpha-\beta} r^{2} \leq|f(z)| \leq r+\frac{1-\beta}{2+\alpha-\beta} r^{2} \\
& 1+\frac{2(1-\beta)}{2+\alpha-\beta} r \leq\left|f^{\prime}(z)\right| \leq 1+\frac{2(1-\beta)}{2+\alpha-\beta} r
\end{aligned}
$$


and the extreme points are

$$
f_{1}(z)=z \text { and } f_{n}(z)=z-\frac{1-\beta}{[n(1+\alpha)-(\alpha+\beta)]} z^{n}, \quad n=2,3, \cdots .
$$

Definition 2.4. For $f(z)=z+\sum_{n=2}^{\infty} a_{n} z^{n}$, let

$$
C P(\alpha)=\left\{f(z) \in S ;\left|\frac{z f^{\prime \prime}(z)}{f^{\prime}(z)}+1-\alpha\right| \leq \operatorname{Re} \frac{z f^{\prime \prime}(z)}{f^{\prime}(z)}+1+\alpha, \quad z \in f, 0<\alpha<\infty\right\} .
$$

Theorem 2.7. If

$$
\sum_{n=2}^{\infty}[n+2(\alpha-1)] n\left|a_{n}\right| \leq 2 \alpha-1
$$

then $f(z)$ of the form (i) is in $C P(\alpha)$.

Proof. From the definition of $C P(\alpha)$, it is sufficient if we see the inequality

$$
\left|1+\frac{z f^{\prime \prime}(z)}{f^{\prime}(z)}-\alpha\right| \leq \operatorname{Re}\left\{\frac{z f^{\prime \prime}(z)}{f^{\prime}(z)}+1+\alpha\right\}
$$

or equivalently

$$
\left|1+\frac{z f^{\prime \prime}(z)}{f^{\prime}(z)}-\alpha\right|-\operatorname{Re}\left\{1+\frac{z f^{\prime \prime}(z)}{f^{\prime}(z)}-\alpha\right\} \leq 2 \alpha
$$

We have

$$
\begin{aligned}
& \left|1+\frac{z f^{\prime \prime}(z)}{f^{\prime}(z)}-\alpha\right|-\operatorname{Re}\left\{1+\frac{z f^{\prime \prime}(z)}{f^{\prime}(z)}-\alpha\right\} \\
\leq & 2\left|1+\frac{z f^{\prime \prime}(z)}{f^{\prime}(z)}-\alpha\right| \\
\leq & 2\left\{\frac{\sum_{n=2}^{\infty} n(n-1)\left|a_{n}\right||z|^{n-1}}{1-\sum_{n=2}^{\infty} n\left|a_{n}\right||z|^{n-1}}+1-\alpha\right\} \\
\leq & 2\left\{\frac{\sum_{n=2}^{\infty} n(n-1)\left|a_{n}\right|}{1-\sum_{n=2}^{\infty} n\left|a_{n}\right|}+1-\alpha\right\} .
\end{aligned}
$$

The last expression is bounded by $2 \alpha$, if and only if (2.6) holds and the proof is complete.

Definition 2.5. Let $C P T(\alpha)$ be the class of functions $f(z)=z-\sum_{n=2}^{\infty} a_{n} z^{n}, a_{n} \geq 0$ such that $f(z)$ is in $C P(\alpha)$.

Since $f(z) \in C P T(\alpha) \Longleftrightarrow z f^{\prime}(z) \in P T(\alpha)$, we get that

$$
P T(\alpha)=\left\{f(z) \in T ;\left|\frac{z f^{\prime}(z)}{f(z)}-\alpha\right| \leq \operatorname{Re} \frac{z f^{\prime}(z)}{f(z)}+\alpha, \quad z \in E, 0<\alpha<\infty\right\} .
$$


Theorem 2.8. $f(z)=z-\sum_{n=2}^{\infty} a_{n} z^{n}, a_{n} \geq 0$ is in $C P T(\alpha)$ if and only if

$$
\sum_{n=2}^{\infty} n(n-1+\alpha) a_{n} \leq \alpha
$$

Proof. From the definition of $C P T(\alpha)$, we get that for $f(z) \in C P T(\alpha)$ and $z$ real,

$$
1-\frac{\sum_{n=2}^{\infty} n(n-1) a_{n} z^{n-1}}{1-\sum_{n=2}^{\infty} n a_{n} z^{n-1}} \geq \frac{\sum_{n=2}^{\infty} n(n-1) a_{n} z^{n-1}}{1-\sum_{n=2}^{\infty} n a_{n} z^{n-1}}+1-2 \alpha
$$

Let $z \rightarrow 1$ along the real axis which gives

$$
\frac{\sum_{n=2}^{\infty} n(n-1) a_{n}}{1-\sum_{n=2}^{\infty} n a_{n}} \leq \alpha
$$

which gives $\sum_{n=2}^{\infty} n[n-1+\alpha] a_{n} \leq \alpha$ and the necessary part follows. The sufficiency part is obvious, because the inequality (2.8) is a better estimate than (2.7) for $f(z)$ to be in $C P T(\alpha)$.

Corollary 2.7. CPT $(\alpha)=C^{*}(1-\alpha)$ for $0<\alpha \leq 1$.

Proof. From Theorem 2.8 we get that

$$
\begin{gathered}
\sum_{n=2}^{\infty} \frac{n(n-1+\alpha)}{\alpha} a_{n} \leq 1 \quad \text { implies that } \\
\sum_{n=2}^{\infty} \frac{n[n-1+\alpha]}{1-(1-\alpha)} a_{n} \leq 1
\end{gathered}
$$

which is in compansion with Theorem $\mathrm{A}$ gives the result.

Corollary 2.8. If $f(z) \in C P T(\alpha)$, then for $0<\alpha \leq 1$

$$
\begin{gathered}
r-\frac{\alpha}{2(1+\alpha)} r^{2} \leq|f(z)| \leq r+\frac{\alpha}{2(1+\alpha)} r^{2} \\
1-\frac{\alpha}{1+\alpha} r \leq\left|f^{\prime}(z)\right| \leq 1+\frac{\alpha}{1+\alpha} r
\end{gathered}
$$

and the extreme points are

$$
f_{1}(z)=z \text { and } f_{n}(z)=z-\frac{\alpha}{n(n-1+\alpha)} z^{n}, \quad n=2,3, \cdots
$$


Proof. The result is immediately available by applying Theorem B and Corollary 2.7 .

Remark. In [5] it is given by

$$
C^{*}(\alpha) \subset T^{*}\left(\frac{2}{3-\alpha}\right)
$$

Therefore

$$
C P T(\alpha) \subset T^{*}\left(\frac{2}{2+\alpha}\right)
$$

In particular

$$
C P T\left(\frac{1}{2}\right) \subset T^{*}\left(\frac{4}{5}\right)
$$

wiht $z-\frac{z^{2}}{6}$ being sharp.

Theorem 2.9. $f(z)=z-\sum_{n=2}^{\infty} a_{n} z^{n}, a_{n} \geq 0$ is in PT( $\left.\alpha\right)$ if and only if

$$
\sum_{n=2}^{\infty}(n+\alpha-1) a_{n} \leq \alpha .
$$

Proof. By Alexander type theorem $f(z) \in C P T(\alpha) \Longleftrightarrow z f^{\prime}(z) \in P T(\alpha)$ therefore we can replace the co-efficient $a_{n}$ in Theorem 2.8 by $a_{n} / n$ which gives the required result.

Corollary 2.9. $P T(\alpha)=T^{*}(1-\alpha), 0<\alpha \leq 1$.

Proof. For $f(z) \in P T(\alpha),(2.9)$ can be written as $\sum_{n=2}^{\infty} \frac{n-(1-\alpha)}{1-(1-\alpha)} a_{n} \leq 1$ which in comparison with Theorem A gives the result.

Thus the distortion theorems and extreme points are easily available by using Corollary 2.9 and Theorem B which we state directly.

Corollary 2.10. Let $f(z) \in P T(\alpha)$. Then for $0<\alpha \leq 1$

$$
\begin{gathered}
r-\frac{\alpha}{1+\alpha} r^{2} \leq|f(z)| \leq r+\frac{\alpha}{1+\alpha} r^{2} \\
1-\frac{2 \alpha}{1+\alpha} r \leq\left|f^{\prime}(z)\right| \leq 1+\frac{2 \alpha}{1+\alpha} r
\end{gathered}
$$

and the extreme points are

$$
f_{1}(z)=z \text { and } f_{n}(z)=z-\frac{\alpha}{n-1+\alpha} z^{n}, n=2,3, \cdots .
$$

Remark. The expression (2.9) gives $f(z) \in P(\alpha)$ if and only if $\sum_{n=2}^{\infty}[n+2(\alpha-$ 1)] $\left|a_{n}\right| \leq 2 \alpha-1$ which coincides with order or growth for co-efficients in $P(\alpha)$, found to be $O\left(\frac{1}{n}\right)$ in [4]. 


\section{Convolution Theorems}

Let $f(z)=z-\sum_{n=2}^{\infty} a_{n} z^{n}, a_{n} \geq 0$ and $g(z)=z-\sum_{n=2}^{\infty} b_{n} z^{n}, b_{n} \geq 0$. We investigate the nature of quasi-convolution $h(z)=f(z) * g(z)=z-\sum_{n=2}^{\infty} a_{n} b_{n} z^{n}$, given that $f(z)$ and $g(z)$ are members of subclasses of $\operatorname{UCV}(\alpha)$ and $S_{p}(\alpha)$.

For $\alpha<-1, S_{p} T(\alpha) \not \subset S$. Therefore we shall consider the case $\alpha>-1$ for $S_{p} T(\alpha)$.

Theorem 3.1. If $f(z)=z-\sum_{n=2}^{\infty} a_{n} z^{n}, a_{n} \geq 0$ and $g(z)=z-\sum_{n=2}^{\infty} b_{n} z^{n}, b_{n} \geq 0$ are elements of $S_{p} T(\alpha)$, then $(f * g)(z)=h(z)=z-\sum_{n=2}^{\infty} a_{n} b_{n} z^{n}$ is in $S_{p} T(\beta)$ where $\beta=\beta(\alpha)=\frac{3-\alpha^{2}}{2(2-\alpha)}, 0 \leq \alpha<1$. The result is best possible.

Proof. By Theorem 2.5, since $f(z)$ and $g(z)$ are in $S_{p} T(\alpha)$ we have

$$
\sum_{n=2}^{\infty}(2 n-1-\alpha) a_{n} \leq 1-\alpha \text { and } \sum_{n=2}^{\infty}(2 n-1-\alpha) b_{n} \leq 1-\alpha
$$

We wish to find largest $\beta=\beta(\alpha)$ such that

$$
\sum_{n=2}^{\infty}(2 n-1-\beta) a_{n} b_{n} \leq 1-\beta
$$

Equivalently we want to show that the conditions

$$
\sum_{n=2}^{\infty} \frac{2 n-1-\alpha}{1-\alpha} a_{n} \leq 1
$$

and

$$
\sum_{n=2}^{\infty} \frac{2 n-1-\alpha}{1-\alpha} b_{n} \leq 1
$$

imply that

$$
\sum_{n=2}^{\infty} \frac{2 n-1-\beta}{1-\beta} a_{n} b_{n} \leq 1
$$

for all $\beta=\beta(\alpha) \leq \frac{3-\alpha^{2}}{2(2-\alpha)}$.

From (3.1) and (3.2) and by means of Cauchy Schwarz inequality, we get that

$$
\sum_{n=2}^{\infty} \frac{2 n-1-\alpha}{1-\alpha} \sqrt{a_{n} b_{n}} \leq 1
$$

It will be sufficient therefore to prove

$$
\frac{2 n-1-\beta}{1-\beta} a_{n} b_{n} \leq \frac{2 n-1-\alpha}{1-\alpha} \sqrt{a_{n} b_{n}}, \beta \leq \beta(\alpha), n=2,3, \cdots .
$$


or

$$
\sqrt{a_{n} b_{n}} \leq\left(\frac{2 n-1-\alpha}{1-\alpha}\right)\left(\frac{1-\beta}{2 n-1-\beta}\right)
$$

From (3.4) it follows that

$$
\sqrt{a_{n} b_{n}} \leq \frac{1-\alpha}{2 n-1-\alpha} \quad \text { for all } n
$$

Inequality (3.5) is equivalent to

$$
\frac{1+\beta}{2} \leq 1-n\left[\frac{1-\alpha}{2 n-1-\alpha}\right]^{2} / 1-\left[\frac{1-\alpha}{2 n-1-\alpha}\right]^{2} .
$$

The right hand side of (3.6) is an increasing function of $n,(n=2,3, \cdots)$; Therefore by setting $n=2$, in (3.6) we get

$$
\frac{1+\beta}{2} \leq \frac{7-\alpha^{2}-2 \alpha}{4(2-\alpha)}
$$

which gives

$$
\beta \leq \beta(\alpha)=\frac{3-\alpha^{2}}{2(2-\alpha)} .
$$

The result is sharp with equality where

$$
f(z)=g(z)=z-\frac{1-\alpha}{3-\alpha} z^{2}
$$

Remark. (i) $\beta(\alpha)=\frac{3-\alpha^{2}}{2(2-\alpha)}>\alpha$ for $0<\alpha<1$.

(ii) For $f(z)$ and $g(z)$ in $S_{p} T(0)$ we have $f(z) * g(z) \in S_{p} T\left(\frac{3}{4}\right)$.

Corollary 3.1. For $f(z)$ and $g(z)$ as in Theorem 3.1 we have

$$
h(z)=z-\sum_{n=2}^{\infty} \sqrt{a_{n} b_{n}} \quad z^{n} \in S_{p} T(\alpha) .
$$

Proof. This result follows from the Cauchy-Schwarz inequality and (3.4). This result is sharp for same function in Theorem 3.1.

Theorem 3.2. For $f(z) \in S_{p} T(\alpha)$ and $g(z) \in S_{p} T(\beta)$ we have

$$
f(z) * g(z) \in S_{p} T\left(\frac{3-\alpha \beta}{4-\alpha-\beta}\right)=S_{p} T(\gamma)
$$

The result is sharp. 
Proof. Proceeding as in the proof of Theorem 3.1 we get

$$
\frac{1+\gamma}{2} \leq \frac{1-n\left(\frac{1-\alpha}{2 n-1-\alpha}\right)\left(\frac{1-\beta}{2 n-1-\beta}\right)}{1-\left(\frac{1-\alpha}{2 n-1-\alpha}\right)\left(\frac{1-\beta}{2 n-1-\beta}\right)}
$$

Right hand side of (3.7) is an increasing function for $n=2,3, \cdots$. Therefore setting $n=2$, we get

$$
\gamma \leq \frac{3-\alpha \beta}{4-\alpha-\beta}
$$

The result is sharp.

Equality is attained for $f(z)=z-\frac{1-\alpha}{3-\alpha} z^{2}$ and $g(z)=z-\frac{1-\beta}{3-\beta} z^{2}$.

Corollary 3.2. Let $f(z), g(z)$ and $h(z) \in S_{p} T(\alpha)$. Then $f(z) * g(z) * h(z) \in S_{p} T(\beta)$ where $\beta=\frac{12-9 \alpha+\alpha^{3}}{13-12 \alpha+\alpha^{2}}$.

Proof. From Theorem 3.1 we get that

$$
f(z) * g(z) \in S_{p} T\left(\frac{3-\alpha^{2}}{4-2 \alpha}\right) .
$$

Therefore

$$
f(z) * g(z) * h(z) \in S_{p} T\left[\frac{3-\alpha\left(\frac{3-\alpha^{2}}{4-2 \alpha}\right)}{4-\alpha-\frac{3-\alpha^{2}}{4-2 \alpha}}\right]=S_{p} T\left[\frac{12-9 \alpha+\alpha^{3}}{13-12 \alpha+\alpha^{2}}\right] .
$$

Now we draw our attention to the class $\operatorname{UCT}(\alpha)$.

Theorem 3.3. Let $f(z)=z-\sum_{n=2}^{\infty} a_{n} z^{n}, a_{n} \geq 0$ and $g(z)=z-\sum_{n=2}^{\infty} b_{n} z^{n}, b_{n} \geq 0$ be elements of $U C T(\alpha)$ then $f(z) * g(z)=h(z)=z-\sum_{n=2}^{\infty} a_{n} b_{n} z^{n} \in U C T(\beta)$ where $\beta=\beta(\alpha) \leq \frac{24-(3+\alpha)^{2}}{(\alpha-5)^{2}-8}$. The result is sharp.

Proof. From Theorem 2.2 we have, for $f(z) \in \operatorname{UCT}(\alpha)$

$$
\sum_{n=2}^{\infty} n(2 n-1-\alpha) a_{n} \leq 1-\alpha
$$

and $g(z) \in \operatorname{UCT}(\alpha)$ implies

$$
\sum_{n=2}^{\infty} n(2 n-1-\alpha) b_{n} \leq 1-\alpha
$$

Therefore by proceeding as in Theorem 3.1 we want to get a $\beta$ satisfying the condition

$$
\sum_{n=2}^{\infty} \frac{n(2 n-1-\beta)}{1-\beta} a_{n} b_{n} \leq 1
$$


Using the same techniques we get that

$$
\frac{1+\beta}{2} \leq \frac{1-\left(\frac{1-\alpha}{2 n-1-\alpha}\right)^{2}}{1-\frac{1}{n}\left(\frac{1-\alpha}{2 n-1-\alpha}\right)^{2}} .
$$

Right hand side of (3.10) is an increasing function of $n(n=2,3, \cdots)$. Therefore by setting $n=2$ we get that

$$
\beta \leq \frac{24-(\alpha+3)^{2}}{(\alpha-5)^{2}-8}
$$

The result is sharp with equality when

$$
f(z)=g(z)=z-\frac{1-\alpha}{2(3-\alpha)} z^{2} .
$$

Remark. For $f(z)$ and $g(z) \in U C T(0)$ we get that

$$
f(z) * g(z) \in U C T\left(\frac{15}{17}\right) .
$$
where

Theorem 3.4. Let $f(z) \in U C T(\alpha)$ and $g(z) \in U C T(\beta)$ then $f(z) * g(z) \in U C T(\gamma)$

The result is sharp.

$$
\gamma=\gamma(\alpha, \beta) \leq \frac{24-(3+\alpha)(3+\beta)}{(5-\alpha)(5-\beta)-8}
$$

Proof. Proceeding as in Theorem 3.3 we get that

$$
\frac{1+\gamma}{2} \leq \frac{1-\left(\frac{1-\alpha}{2 n-1-\alpha}\right)\left(\frac{1-\beta}{2 n-1-\beta}\right)}{1-\frac{1}{n}\left(\frac{1-\alpha}{2 n-1-\alpha}\right)\left(\frac{1-\beta}{2 n-1-\beta}\right)}
$$

Right hand side of (3.11) is an increasing function for $n=2,3, \cdots$. Therefore setting $n=2$ we get that

$$
\gamma=\gamma(\alpha, \beta) \leq \frac{24-(3+\alpha)(3+\beta)}{(5-2)(5-\beta)-8}
$$

which is the required result. The result is sharp. Equality is attained when $f(z)=$ $z-\frac{1-\alpha}{2(3-\alpha)} z^{2}$ and $g(z)=z-\frac{1-\beta}{2(3-\beta)} z^{2}$.

Theorem 3.5. Let $f(z)=z-\sum_{n=2}^{\infty} a_{n} z^{n}, a_{n} \geq 0$ and $g(z)=z-\sum_{n=2}^{\infty} b_{n} z^{n}, b_{n} \geq 0$ be in $S_{p} T(\alpha, \beta)$. Then $(f * g)(z)=h(z)=z-\sum_{n=2}^{\infty} a_{n} b_{n} z^{n} \in S_{p} T(A, B)$ where

$$
\begin{gathered}
A=A(\alpha, \beta)=2\left[1-\frac{\alpha+\beta}{1+\alpha}\right] \\
B=B(\alpha, \beta)=\frac{\alpha-\beta}{1+\alpha}\left[2-\frac{\alpha+\beta}{1+\alpha}\right] .
\end{gathered}
$$


The result is sharp.

Proof. From Theorem 2.6 we get that $f(z) \in(\alpha, \beta)$ give

$$
\sum_{n=2}^{\infty}[n(1+\alpha)-(\alpha+\beta)] a_{n} \leq 1-\beta
$$

and $g(z) \in S_{p} T(\alpha, \beta)$ gives

$$
\sum_{n=2}^{\infty}[n(1+\alpha)-(\alpha+\beta)] b_{n} \leq 1-\beta
$$

We like to find the largest $\varepsilon$ and $\delta$ such that

$$
\sum_{n=2}^{\infty}[n(1+\varepsilon)-(\varepsilon+\delta)] a_{n} b_{n} \leq 1-\delta .
$$

By using the same techniques as in previous theorems we get that, it is enough if we show

$$
\left[\frac{1-\beta}{n(1+\alpha)-(\alpha+\beta)}\right] \leq\left[\frac{n(1+\alpha)-(\alpha+\beta)}{1-\beta}\right]\left[\frac{1-\delta}{n(1+\varepsilon)-(\varepsilon+\delta)}\right] \text { for all } n
$$

which is equivalent to show that

$$
\frac{\varepsilon+\delta}{1+\varepsilon} \leq \frac{1-n\left[\frac{1-\beta}{n(1+\alpha)-(\alpha+\beta)}\right]^{2}}{1-\left[\frac{1-\beta}{n(1+\alpha)-(\alpha+\beta)}\right]^{2}} .
$$

Right hand side of the above expression is an increasing function for $n=2,3, \cdots$.

Therefore by setting $n=2$, we get

$$
\frac{\varepsilon+\delta}{1+\varepsilon} \leq \frac{2\left[1-\left(\frac{\alpha+\beta}{1+\alpha}\right)\right]+\left(\frac{\alpha+\beta}{1+\alpha}\right)\left[2-\left(\frac{\alpha+\beta}{1+\alpha}\right)\right]}{1+2\left[1-\frac{\alpha+\beta}{1+\alpha}\right]}
$$

which gives the required result. The result is sharp with equality when

$$
f(z)=g(z)=z-\left(\frac{1-\beta}{2+\alpha-\beta}\right) z^{2} .
$$

Now we show that the class $P T(\alpha)$ is not closed under convolution.

Let $f(z)=z-\sum_{n=2}^{\infty} a_{n} z^{n}, a_{n} \geq 0 \in P T(\alpha)$ and $g(z)=z-\sum_{n=2}^{\infty} b_{n} z^{n}, b_{n} \geq 0 \in$ $P T(\alpha)$.

Now we are interested to find if $h(z)=z-\sum_{n=2}^{\infty} a_{n} b_{n} z^{n}$ is possible to be in $P T(\beta)$ for some $\beta$.

Equivalently by using Theorem 2.9 we are interested in finding the existence of the condition

$$
\sum_{n=2}^{\infty}(n+\beta-1) a_{n} b_{n} \leq \beta
$$


for

and

$$
\sum_{n=2}^{\infty}(n+\alpha-1) a_{n} \leq \alpha
$$

$$
\sum_{n=2}^{\infty}(n+\alpha-1) b_{n} \leq \alpha
$$

(3.16) and (3.17) together imply with Cauchy Schwarz inequality that

$$
\sum_{n=2}^{\infty}(n-1+\alpha) \sqrt{a_{n} b_{n}} \leq 1 .
$$

Then by using the same techniques we are able to get

$$
\beta \leq \frac{\alpha^{2}}{(1-n)(2 \alpha+n-1)} \quad \text { for all } n .
$$

Right hand side of the above expression is an increasing function for $n=2,3, \cdots$. Therefore by setting $n=2$, gives

$$
\beta \leq \frac{-\alpha^{2}}{2 \alpha+1}
$$

Since, $0<\alpha<\infty$ for $f(z) \in P T(\alpha)$ we get that $\beta$ in (3.18) satisfies the condition $-\infty<\beta<0$ which gives that $f(z) * g(z) \notin P(\beta)$ for any $\beta$.

\section{Acknowledgement}

We thank the referee for his useful comments.

\section{References}

[1] A. W. Goodman, "On uniformly convex functions," Ann. Polon. Maht., 56 (1991), 87-92.

[2] F. Ronning, "On starlike functions associated with parabolic regin," Ann. Univ. Marie. Curie Sklodowska Sect. A., Vol. XIV. 14 (1991), 117-122.

[3] F. Ronning, "Uniformly convex functions and a corresponding class of starlike functions," Proc. Amer. Math. Soc., 18(1), May (1993), 189-196.

[4] F. Ronning, "Integral representations for bounded starlike functions," Annales Polon. Math., 60 (1995), 289-297.

[5] H. Silverman, "Univalent functions with negative co-efficients," Proc. Amer. Math. Soc., 51(1), Aug. (1975), 109-116.

[6] H. Silverman, "Convolutions of univalent functions with negative co-efficients," Ann. Univ. Marie. Curie Sklodoska Sect. A., Vol. XXIX, 12 (1975), 99-107.

Department of Mathematics, Loyola College, Madras-600 034, India.

Ramanujan Institute, University of Madras, Madras- 600 005, India.

Department of Mathematics, Loyola College, Madras- 600 034, India. 